\title{
Prevalence of insulin resistance in patients with polycystic ovary syndrome referred to Sarem Hospital in 2020-2021
}

\section{ARTICLE INFO}

Article Type

Original article

\section{Authors}

Fatemeh Jalalizand ${ }^{1}$,MD,

AboTaleb Saremi 2* iD, MD

Homa Bahrami ${ }^{1}, \mathrm{MD}$,

Fariba Mohammad Aliha, BCs

${ }^{1}$ Sarem Fertility \& Infertility Research Center (SAFIR), Sarem Women's Hospital, Iran University of Medical Sciences (IUMS), Tehran, Iran.

2 Sarem Fertility \& Infertility Research Center (SAFIR) \& Sarem Cell Research Center (SCRC), Sarem Women's Hospital, Iran University of Medical Sciences (IUMS),

Tehran, Iran.

*Corresponding Author

Address: Sarem Fertility \& Infertility Research Center (SAFIR) \& Sarem Cell Research Center (SCRC), Sarem Women's Hospital, Tehran, Iran.

Phone: +21 44670883

Dr.saremi@sarem.org

\section{ABSTRACT}

Introduction: Polycystic ovary syndrome (PCOS) is one of the most common hyperandrogenic disorders in the fertility age. In patients suffering from this disease, etiologies and clinical manifestations such as insulin resistance, hyperinsulinemia, and hyperandrogenism of insulin resistance are seen. According to statistics, about 50 to 70 percent of women with this syndrome have different degrees of insulin resistance that it is most important in term of common health of these subjects. The aim of this study was to evaluate the prevalence of insulin resistance in a number of patients with PCOS referred to Sarem Hospital in 20202021.

Martial and methods: This study was a descriptive cross-sectional study with a convenience sampling method among PCOS patients with an age range of 27 to 30 years referred to the infertility special clinic. Collected data were analyzed using descriptive statistics with SPSS software version 19.

Results: A total of 162 subjects with PCOS in sonography were entries in this study. The test of insulin resistance evaluation was performed in a fixed method in 87 of them and the rest were excluded from research due to incomplete records. In this research, the prevalence of insulin resistance was $69.3 \%$.

Conclusion: The prevalence of insulin resistance in patients with polycystic ovary syndrome in this study was almost consistent with the maximum of presented statistics in previous studies and it is necessary to consider this point in the treatment of these patients.

Keywords: Polycystic Ovary Syndrome (PCOS); Insulin Resistance; Hyperinsulinemia; Hyperandrogenism; Anovulation.

\section{Article History}

Received: January 19, 2021

Accepted: February 03, 2021

e Published: August 12, 2021

Copyright(C) 2021, ASP Ins. This open-access article is published under the terms of the Creative Commons Attribution-Noncommercial 4.0 International License which permits Share (copy and distribute the material in any medium or format) and Adapt (remix, transform, and build 
عدم تكميل برونده از مطالعه حذف گرديدند. در اين بزروهش، شيوع مقاومت

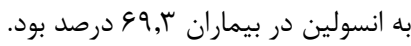

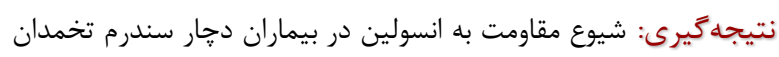

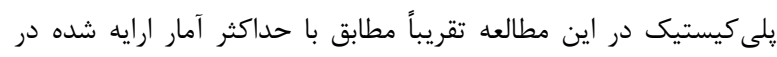

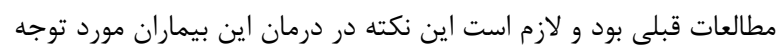
قرار زيرد. - مطات

كليد وارزها: سندرم تخمدان يلىكيستيك؛ مقاومت به انسولين؛ هييرانسولينمى؛ هيير آندروزنيسه؛ عدم تخمك كذارى.

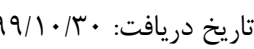

تاريخ بذيرش:

"نويسنده مسئول: ابوطالب صارمى : تاريخ

مقدمه

عدم تخمك كذارى يك عارضه شايع با تظاهرات بالينى متفاوت شامل آمنوره، اليكومنوره، خونريزى غير طبيعى رحمى و ورى هيرسوتيسمه است.

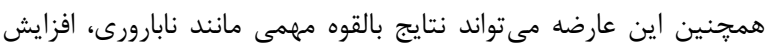

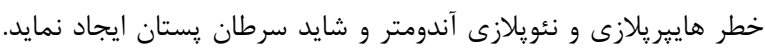

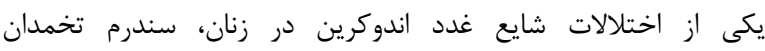

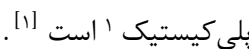

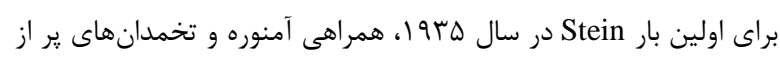

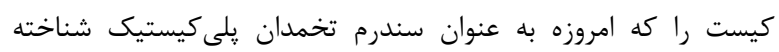

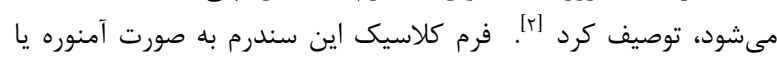

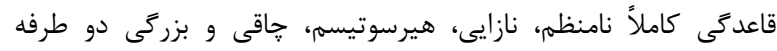

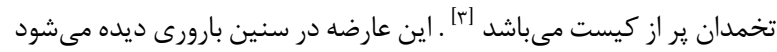

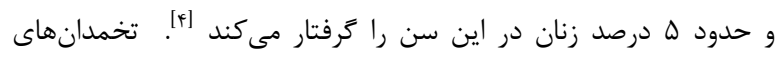

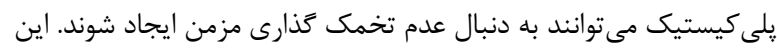

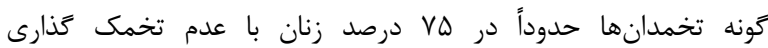
ديده مىشوند [هـ] امروزه محققان معتقدند علت سندرم تخمدان يلى كيستيك ناشى از عوامل

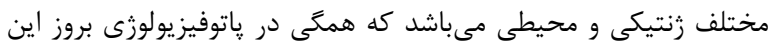

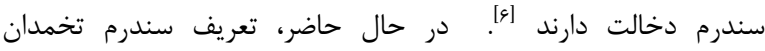

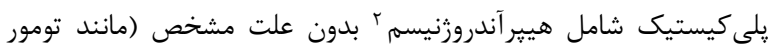

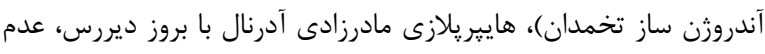

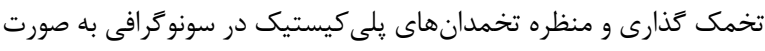

Hyperandrogenism

\section{شيوع مقاومت به انسولين در بيماران}

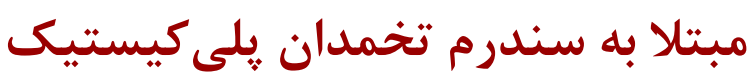

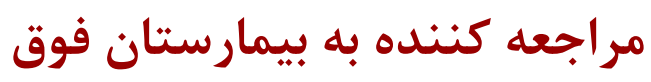

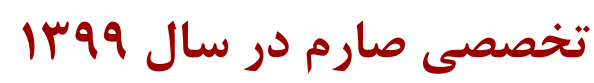

فاطمه جلالى زند ' ، ابوطالب صارمى r* iD )، هما بهرامى'، فريبا محمد عليها

' مركز تحقيقات بارورى و نابارورى صارم، بيمارستان فوق تخصصى صارم،

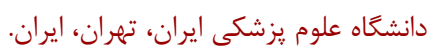

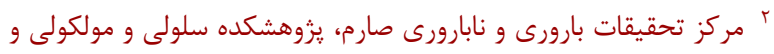

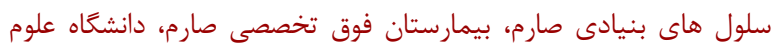
يزشكى ايران، تهران، ايران.

جكيده

مقدمه: سندرم تخمدان يلىسيستيك يك از شايعترين اختلالات

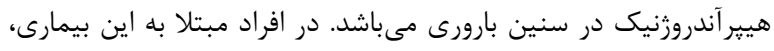

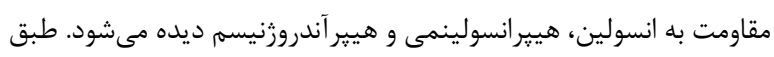

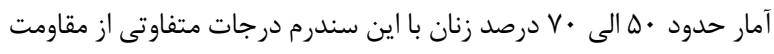

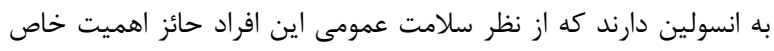

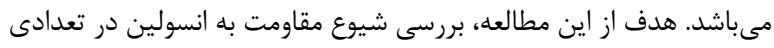

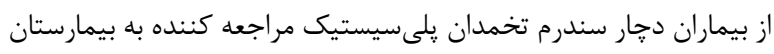

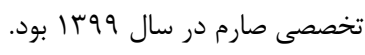
مواد و روش ها: اين مطالعه يك يروهش توصيفى-مقطعى است كه در در

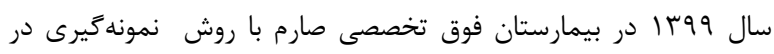

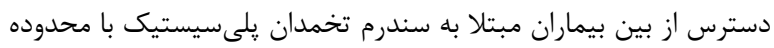

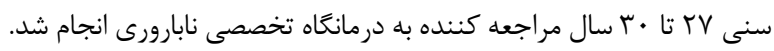

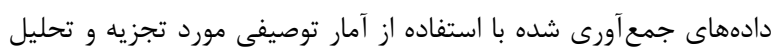
قرار ترفتند. يافته ها: تعداد كاد أن نفر بيمار كه سندرم تخمدان يلىسيستيك در

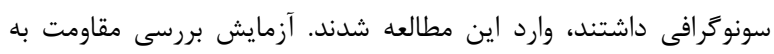

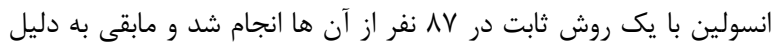

Polycystic Ovary Syndrome (PCOS)

دانشنامه صارم در طب بارورى

دوره ه، شماره f) - زمستان 
انستيتو تحقيقات صارم رسيد و از كليه شركت كنندكان قبل از شروع مطالعه، رضايتنامه كتبى اخذ كرديد.

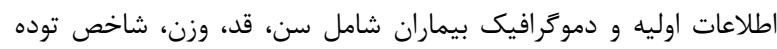

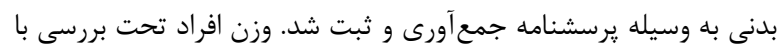

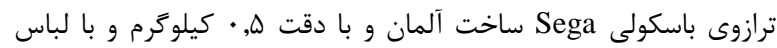

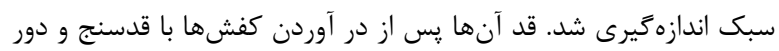

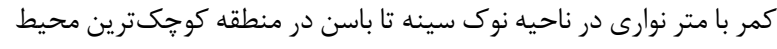

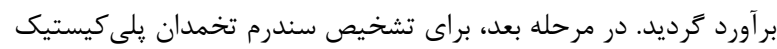

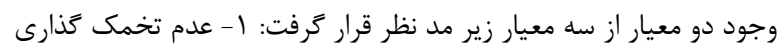

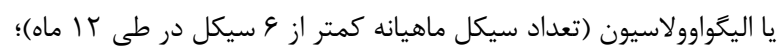

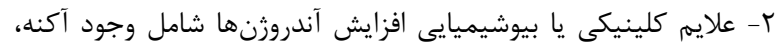

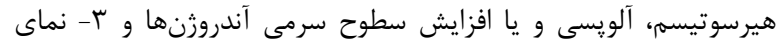

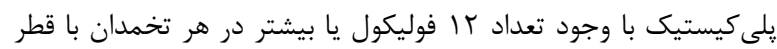

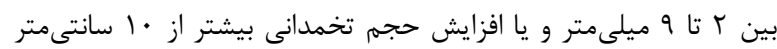
مكعب [if (1+]

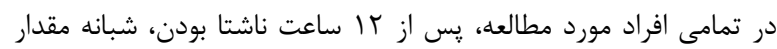
ه سىسى خون وريدى براى بررسى قند خون ناشتا، سطح انسولين و

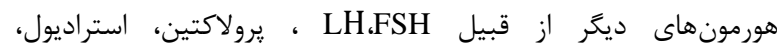

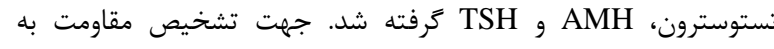

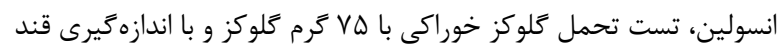

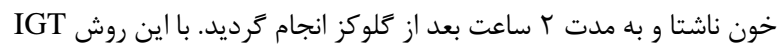

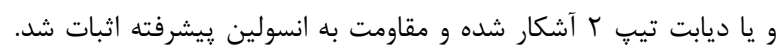

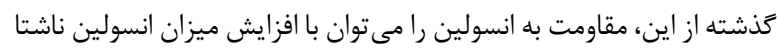

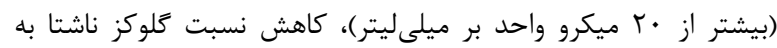

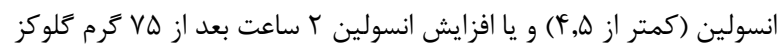

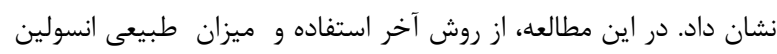

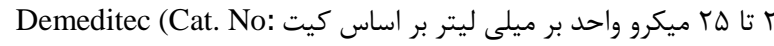
DE2935)

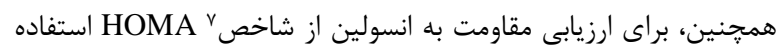

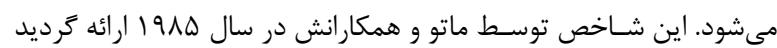

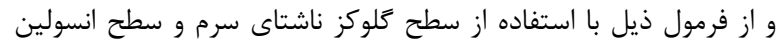

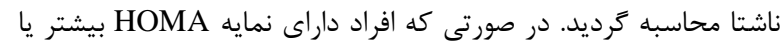

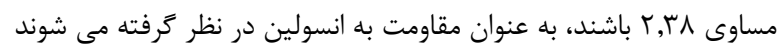
[10.19]

HOMA-IR $=[$ Fasting Glucose $(\mathrm{mg} / \mathrm{dl}) \times$ Fasting Insulin $(\mu \mathrm{U} / \mathrm{ml}) / 22.5]$

با اين حال، ملاك يا مطالعه اثبات مفيد بودن اين آزمايشات جهت تشخيص

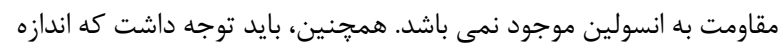

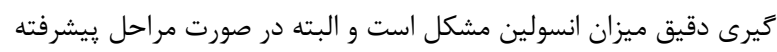

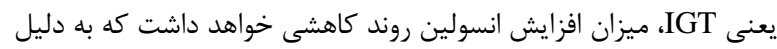

بيش از هشت فوليكول با اندازه Y تا 1 ميليمتر و افزايش استروماى تخمدان

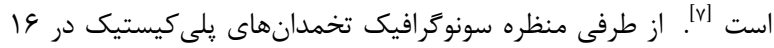

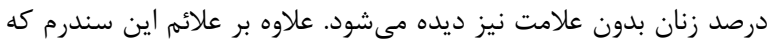

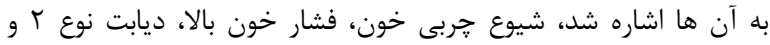

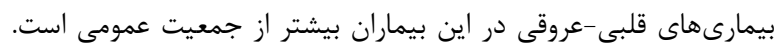

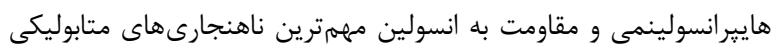

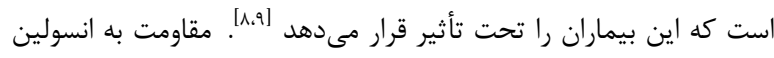
به وضعيتى كفته مى شود كه با يك مقدار مشخص انسولين، كاهش كمار كمتر

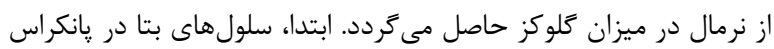

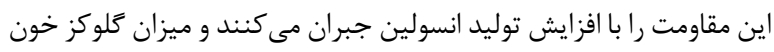

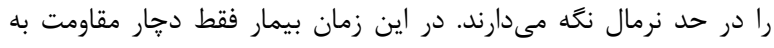

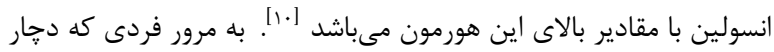

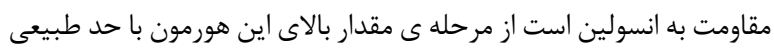

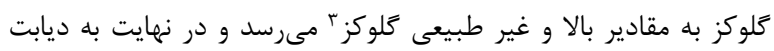

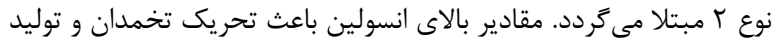

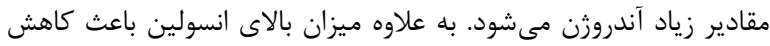

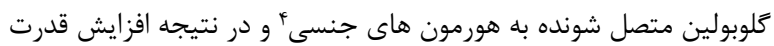

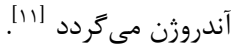
احتمال دارد كه مقادير بالاى انسولين در سطح مغز نيز باعث افزايش ترشح

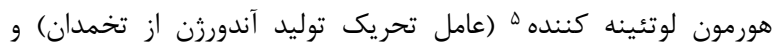

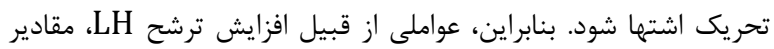

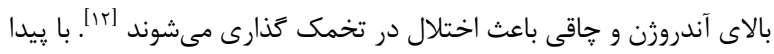

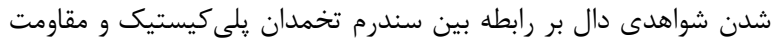

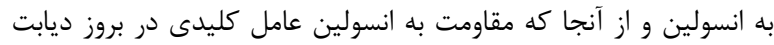

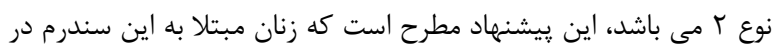

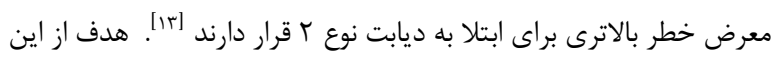

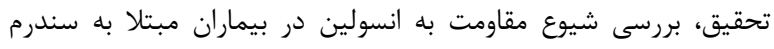

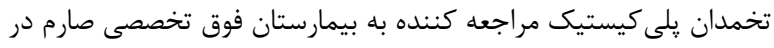

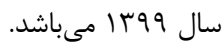

\section{مواد و روش ها}

در اين مطالعه توصيفى -مقطعى \& كه در سال 99 در بيمارستان صارم انجام

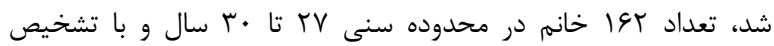

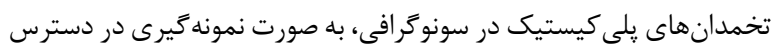

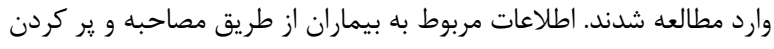

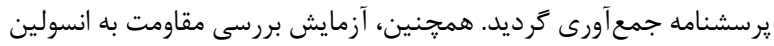

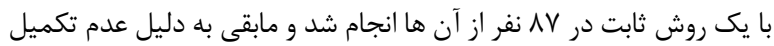

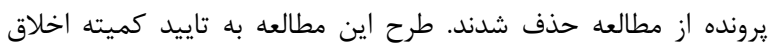

Impaired Glucose Tolerance (IGT) Sex Hormone Binding Globulin (SHBG) Luteinizing Hormone (LH)

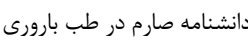

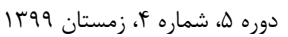


جدول شماره ז: مقادير سونوكر افيك و آزمايشكاهى بيماران مورد مطالعه

\begin{tabular}{|c|c|c|c|c|}
\hline حدائزي. & حاقال & إنحراف ميارا & مبائين & 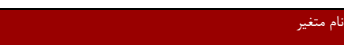 \\
\hline & 6 & 4.93 & 13.67 & حجم تخدان راست (سانتميتر مكب) \\
\hline 25 & 10 & 3.92 & 13.87 & تعداد فوليكول در تخمدان راست \\
\hline 9 & 4.5 & 0.99 & 6.49 & 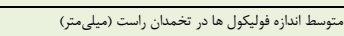 \\
\hline 27 & 5 & 4.48 & 12.24 & حجم تخمدان جب (سانتممتر مكبب) \\
\hline 25 & 10 & 3.56 & 13.27 & تعداد فوليكول در تخمدان جبي \\
\hline 9 & 4 & 0.98 & 6.63 & متوتط اندازه فوليكول ها در تخمان جب (ميلمترت) \\
\hline 11.7 & 2.3 & 1.99 & 5.54 & ضخامت أندومتر (ميلىمتر) \\
\hline 185 & 72 & 16.38 & 94.38 & قتد خون ناشتا (ميلى كرم بر دسىليتر) \\
\hline 230 & 77 & 23.54 & 108.3 & 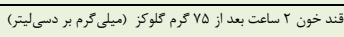 \\
\hline 166 & 1.2 & 2079 & 16.53 & انسولين ناشتا (ميلىواحد بر ميلىليتر) \\
\hline 150 & 2.3 & 31.68 & 43.71 & انسولين ب ساعت بعدا ز هل كرم ملوكز (ميليواحد بر ميلى ليتر) \\
\hline 11 & 2 & 1.84 & 6.4 & FSH \\
\hline 26.2 & 1.6 & 4.93 & 9.19 & LH \\
\hline 78 & 1.6 & 17.11 & 44.82 & استراديول (ييكوكرم بر ميلى ليتر) \\
\hline 969 & 4.7 & 177.03 & 69.42 & ي برولالتين (ناوكرم بر ميلى ليتر) \\
\hline 11.6 & 2 & 1.89 & 2.56 & (مSH TSH \\
\hline 334 & 0.1 & 49.49 & 9.83 & تستوسترون (ييكوكرم بر ميلىليتر) \\
\hline 15.3 & 1 & 3.57 & 5.84 & AMH \\
\hline
\end{tabular}

مسخصص مىشود. بيماران با اين سندرم بـا مـشكلات نازايى در سنين

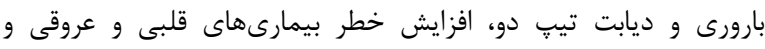

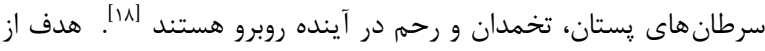

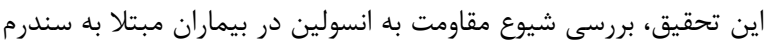

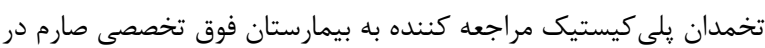

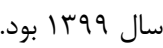

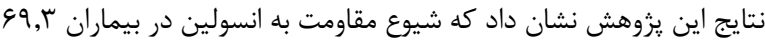

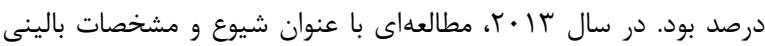

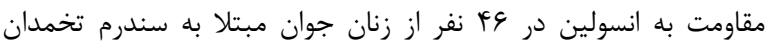

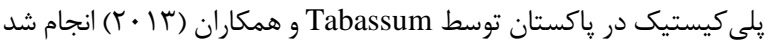

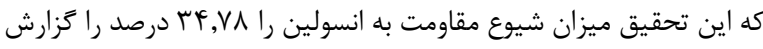
كرد. ميانگين سنى اين افراد سنى مطالعه ما نزديك بود.

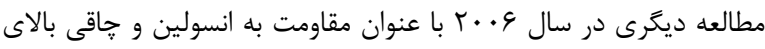

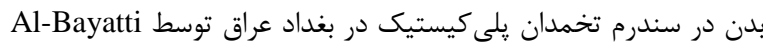

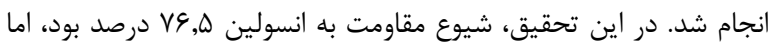

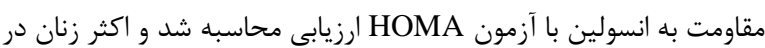

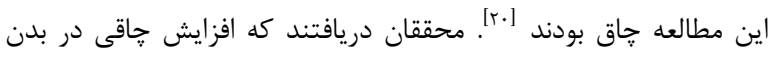

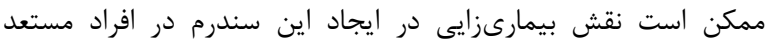

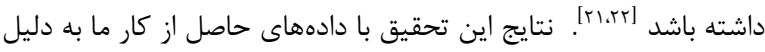

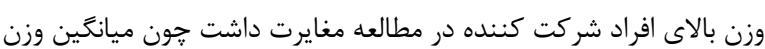

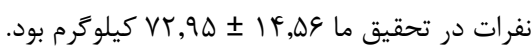

فرسودحى سلول هاى بتا يانكراس مىباشد. ضمناً، مقاومت به انسولين از نظر

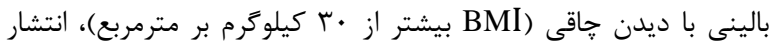

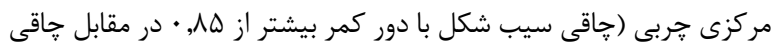

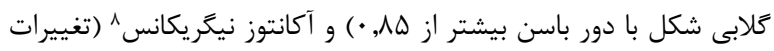

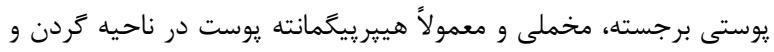

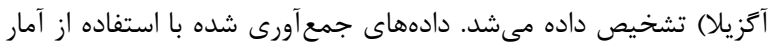

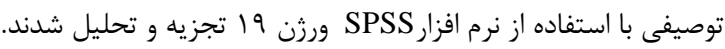

\section{هافته ها}

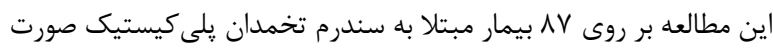

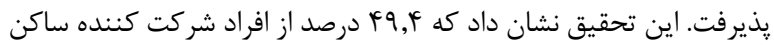

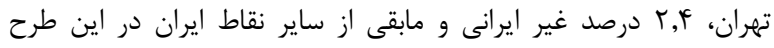

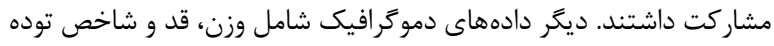

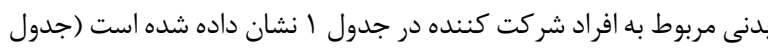

جدول ا: مشخصات دموكرافيك تعداد NV نفر بيمار مبتلا به سندرم تخمدان

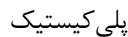
ميانكَين 土 انحراف معيار يارامترها

\begin{tabular}{|c|c|}
\hline $72.95 \pm 4.56$ & وزن (كيلوكرم) \\
\hline $162.1 \pm 6.16$ & قد (سانتىمتر) \\
\hline $27.54 \pm 2.25$ & شاخص توده بدنى (كيلو كرم بر مترمربع) \\
\hline
\end{tabular}

علايم سونوگرافيك شامل حجم و اندازه تخمدانها، تعداد فوليكولها و

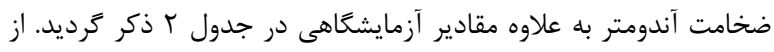

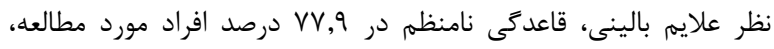

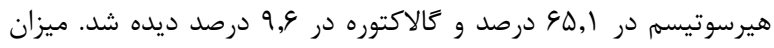

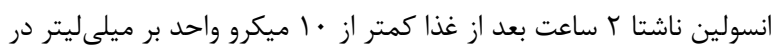

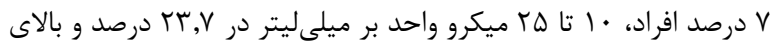

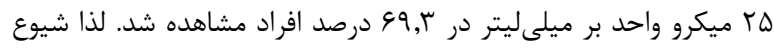

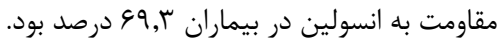

\section{بحث}

بيمارى تخمدان يلى كيستيك يكى از شايعترين اخـتلالات غدد داخلى در

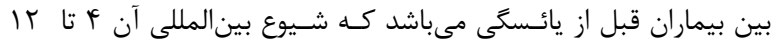

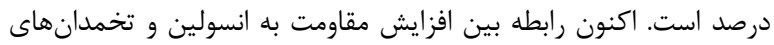

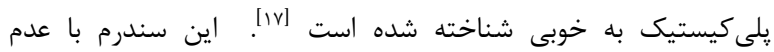

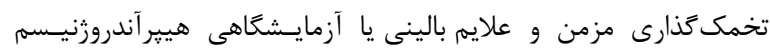

Acanthosis Nigricans ${ }^{\wedge}$ 
بايد مقاومت به انسولين در آنها شناسايى شود تا به موقع از بروز و پيشرفت

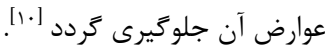

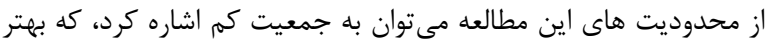

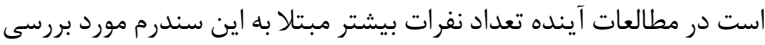

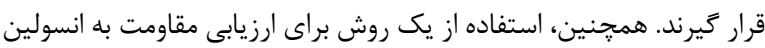

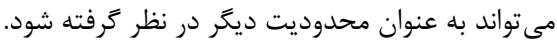

نتيجه كَيرى

هدف از اين مطالعه حاضر، بررسى شيوع مقاومت به انسولين در بيماران

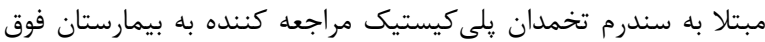

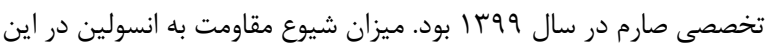

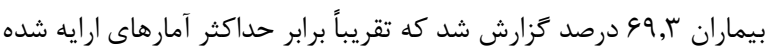

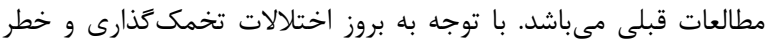

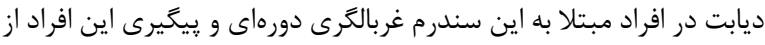

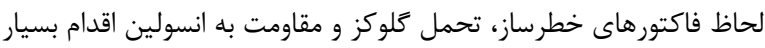

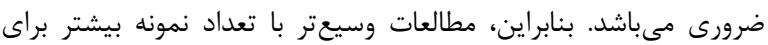

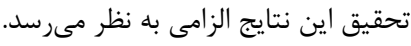

از لطف بى دريغ كاركنان محترم بيمارستان تخصصى صارم و انستيتو تحقيقات صارم و همجنين شركت كنند

\section{ملاحظات اخلاقى:}

كليه دادهاى اين مطالعه محرمانه بوده و بيماران از مراحل ماحل درمان آنان آحاه

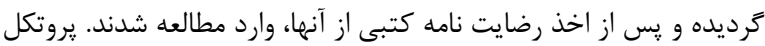
يزوهش توسط "كميته تحقيق و اخلاق" انستيتو تحقيقات صارم تأييد شد. أندا.

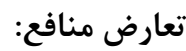

در اين مطالعه تعارض منافع وجود نداشت.

هزينهاى يزوهش توسط انستيتو تحقيقات صارم تامين شده است.

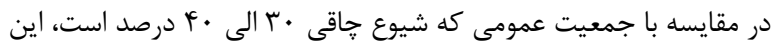

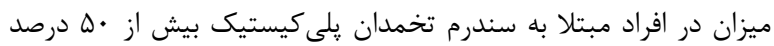

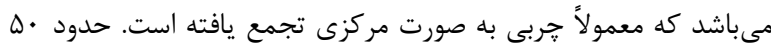

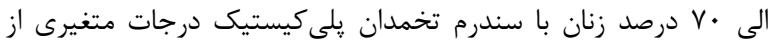

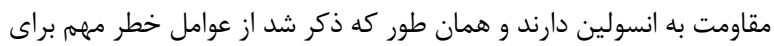

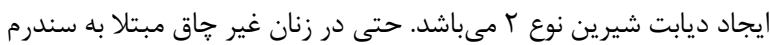

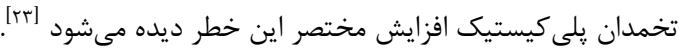

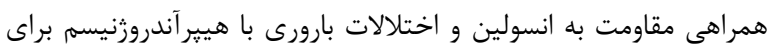

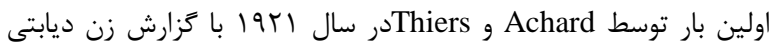

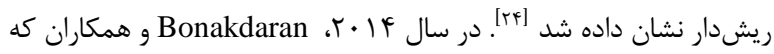

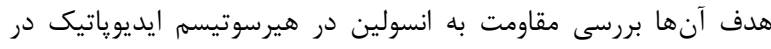

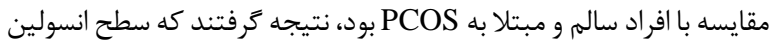

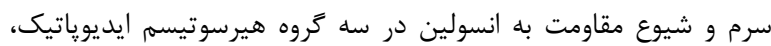
PCOS

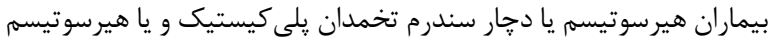

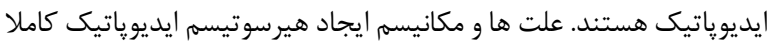

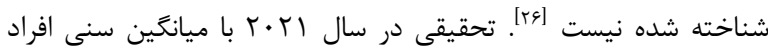

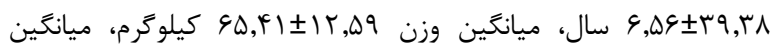

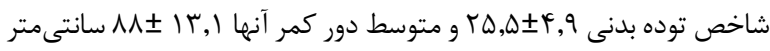

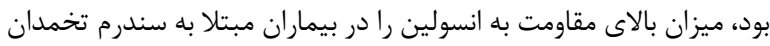

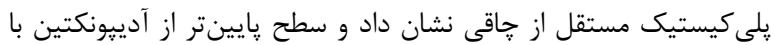

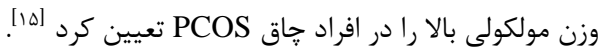

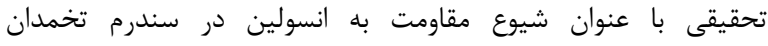

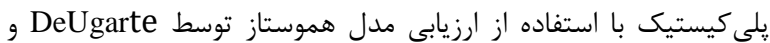

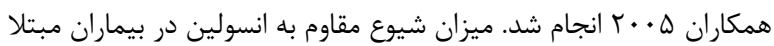

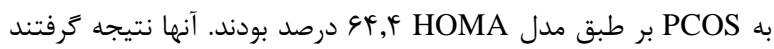
كه بيماران با مقاومت به انسولين بيشتر از نظر بالينى تحت تأثير قرار

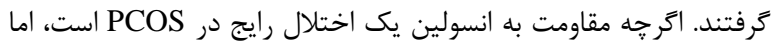

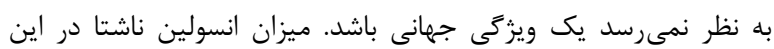

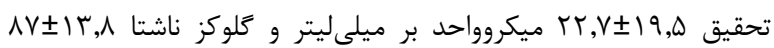

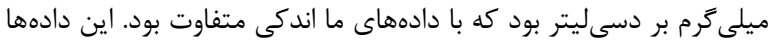

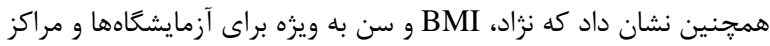

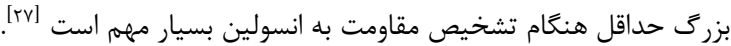

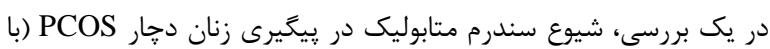

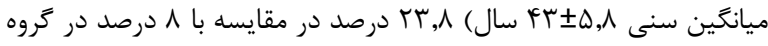

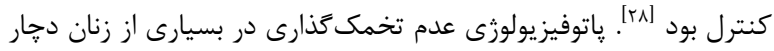

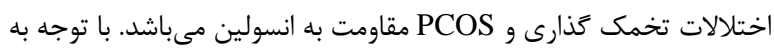

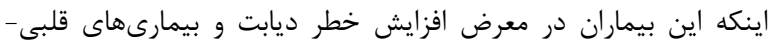

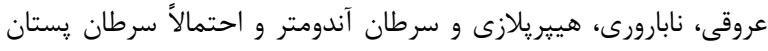

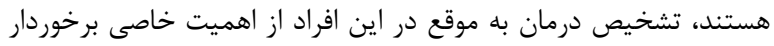

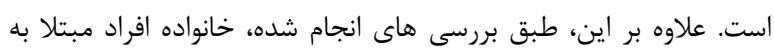

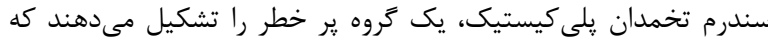


[12] L. Asarian, N. Geary, Modulation of appetite by gonadal steroid hormones, Philos. Trans. R. Soc. B Biol. Sci. 361 (2006) 1251-1263.

[13] M.T. Sheehan, Polycystic ovarian syndrome: diagnosis and management, Clin. Med. Res. 2 (2004) 13-27.

[14] M. Mortensen, D.A. Ehrmann, E. Littlejohn, R.L. Rosenfield, Asymptomatic volunteers with a polycystic ovary are a functionally distinct but heterogeneous population, J. Clin. Endocrinol. Metab. 94 (2009) 1579-1586.

[15] F.K.H. Shirazi, Z. Khodamoradi, M. Jeddi, Insulin resistance and high molecular weight adiponectin in obese and non-obese patients with Polycystic Ovarian Syndrome (PCOS), BMC Endocr. Disord. 21 (2021) 1-7.

[16] O.O. Sonuga, F.M. Abbiyesuku, K.S. Adedapo, A.A. Sonuga, Insulin resistance index and proatherogenic lipid indices in the offspring of people with diabetes, Int. J. Diabetes Metab. 25 (2019) 11-18.

[17] D.A. Ehrman, R.B. Barnes, R.L. Rosenfield, Polycystic ovary syndrome as a form of functional ovarian hyperandrogenism due to dysregulation of androgen secretion, Endocr. Rev. 16 (1995) 322-353.

[18] F. MIRZAEI, N. Kazemi, STUDY THE PREVALENCE OF POLYCYSTIC OVARIAN SYNDROME IN WOMELF WITH TYPE 2 DIABETES REFERRING TO KERMAN DIABETES CLINIC, (2008).

[19] R. Tabassum, F. Imtiaz, S. Sharafat, Prevalence and clinical profile of insulin resistance in young women of poly cystic ovary syndrome: A study from Pakistan, Pakistan J. Med. Sci. 29 (2013) 593.

[20] A.A. Al-Bayatti, Insulin resistance and upperbody obesity in polycystic ovary syndrome, Middle East Fertil. Soc. J. 11 (2006) 202.

[21] A. Dunaif, Insulin action in the polycystic ovary syndrome, Endocrinol. Metab. Clin. North Am. 28 (1999) 341-359.

[22] A. Dunaif, K.R. Segal, W. Futterweit, A. Dobrjansky, Profound peripheral insulin resistance, independent of obesity, in polycystic ovary syndrome, Diabetes. 38 (1989) 11651174.

[23] R. Benítez, T. Petermann, A. Palomino, B. Angel, M. Maliqueo, F. Pérez, M. Calvillan, Prevalence of metabolic disorders among family members of patients with polycystic ovary syndrome, Rev. Med. Chil. 129 (2001) 707-712.

[24] C. Achard, J. Thiers, Le virilisme pilaire et son

\section{منابع}

[1] L. Speroff, M.A. Fritz, Clinical gynecologic endocrinology and infertility, lippincott Williams \& wilkins, 2005.

[2] I.F. Stein, Amenorrhea associated with bilateral polycystic ovaries, Am J Obs. Gynecol. 29 (1935) 181-191.

[3] R.E.P.C.W. Group, Revised 2003 consensus on diagnostic criteria and long-term health risks related to polycystic ovary syndrome (PCOS), Hum. Reprod. 19 (2004) 41-47.

[4] E. Diamanti-Kandarakis, A. Dunaif, Insulin resistance and the polycystic ovary syndrome revisited: an update on mechanisms and implications, Endocr. Rev. 33 (2012) 981-1030.

[5] R. Pasquali, Contemporary approaches to the management of polycystic ovary syndrome, Ther. Adv. Endocrinol. Metab. 9 (2018) 123-134.

[6] E. Diamanti-Kandarakis, C. Piperi, G. Argyrakopoulou, J. Spina, L. Papanastasiou, A. Bergiele, D. Panidis, Polycystic ovary syndrome: the influence of environmental and genetic factors, HORMONES-ATHENS-. 5 (2006) 17.

[7] R.L. Rosenfield, D.A. Ehrmann, The pathogenesis of polycystic ovary syndrome (PCOS): the hypothesis of PCOS as functional ovarian hyperandrogenism revisited, Endocr. Rev. 37 (2016) 467-520.

[8] M. Ebrahimi-Mamaghani, M. Saghafi-Asl, S. Pirouzpanah, A. Aliasgharzadeh, S. Aliashrafi, N. Rezayi, M. Mehrzad-Sadaghiani, Association of insulin resistance with lipid profile, metabolic syndrome, and hormonal aberrations in overweight or obese women with polycystic ovary syndrome, J. Health. Popul. Nutr. 33 (2015) 157.

[9] Z. Zahiri, S.H. Sharami, F. Milani, F. Mohammadi, E. Kazemnejad, H. Ebrahimi, S.F.D. Heirati, Metabolic syndrome in patients with polycystic ovary syndrome in Iran, Int. J. Fertil. Steril. 9 (2016) 490.

[10] M. Akbarzadeh, F. Moradi, M.H. Dabbaghmaneh, M.E. Parsanezhad, P. Jafary, Insulin Resistance in First Degree Relatives of Patients with Polycystic Ovarian Syndrome, (2010).

[11] C.G. Baptiste, M.-C. Battista, A. Trottier, J.-P. Baillargeon, Insulin and hyperandrogenism in women with polycystic ovary syndrome, J. Steroid Biochem. Mol. Biol. 122 (2010) 42-52. 
شيوع مقاومت به انسولين در بيماران مبتلا به سندرم تخمدان يلى كيستيك مراجعه كننده به بيمارستان فوق تخصصى صارم

association a l'insuffisance glycolytique (diabete des femmes a barbe), Bull Acad Natl Med. 86 (1921) 51-66.

[25] S. Bonakdaran, F. Barazandeh Ahmadabadi, Assessment of insulin resistance in idiopathic hirsutism in comparison with Polycystic Ovary Syndrome (PCOS) patients and healthy individuals, Med. J. Mashhad Univ. Med. Sci. 56 (2014) 340-346.

[26] R. Azziz, L.A. Sanchez, E.S. Knochenhauer, C. Moran, J. Lazenby, K.C. Stephens, K. Taylor, L.R. Boots, Androgen excess in women: experience with over 1000 consecutive patients, J. Clin. Endocrinol. Metab. 89 (2004) 453-462.

[27] C.M. DeUgarte, A.A. Bartolucci, R. Azziz, Prevalence of insulin resistance in the polycystic ovary syndrome using the homeostasis model assessment, Fertil. Steril. 83 (2005) 1454-1460.

[28] M. Hudecova, J. Holte, M. Olovsson, A. Larsson, C. Berne, I. Sundstrom-Poromaa, Prevalence of the metabolic syndrome in women with a previous diagnosis of polycystic ovary syndrome: longterm follow-up, Fertil. Steril. 96 (2011) 12711274. 